\title{
Modifications of lampbrush chromosome structure of the European domestic goose Anser anser
}

\author{
Katarzyna Andraszek and Elżbieta Smalec \\ Department of Animal Genetics and Horse Breeding, Institute of Bioengineering and Animal Breeding, University of \\ Life Sciences and Humanities, Siedlce, Poland
}

\begin{abstract}
Lampbrush chromosomes (LBCs) represent a new model in avian cytogenetics and are increasingly more often used in poultry chromosome analyses. Additionally, lampbrush chromosomes are considered as model structures in the study of transcription regulation. Changes in transcription activity are reflected as modifications of LBC morphological structure and associated with physiological processes in the organism. The aim of the present study was to compare transcriptional activity of the first five lampbrush macrochromosomes and ZW sex lampbrush bivalents sampled from the oocytes of geese prior to and after the reproductive period. The respective bivalents sampled before and after reproduction have similar sizes but differ in morphological structure. Side loops of lampbrush chromosomes are sites of transcription activity. The activity varies according to the loop size. As the loops become more prominent, the activity grows and vice versa. Lampbrush chromosomes sampled after reproduction have smaller side loops. On the other hand, inactive chromomeres become prominent in the chromosomes. Marker loops are the last structures to be degraded after the end of reproduction. Consequently, they are used for identifying particular bivalents at different stages of cellular transcriptional activity.
\end{abstract}

Keywords: Anser anser goose, reproduction, meiotic chromosome, lampbrush chromosome, transcription

\section{Introduction}

High transcriptional activity observed in avian oocytes during previtellogenesis causes chromatin decondensation. Elongated and decondensed chromosomal forms appear. These are lampbrush chromosomes ( $\mathrm{LBCs}$ ) with thick mantles of side loops that constitute the transcription units. Each LBC consists of alternately positioned chromomeres (condensed chromatin) with one pair or numerous pairs of loops (transcriptionally active chromatin) (Gaginskaya et al. 1984, Callan 1986, MacGregor 1986, Morgan 2002, 2007, Austin et al. 2009). Lampbrush chromosomes were introduced as an object of cytogenetic study of poultry by Kroptova \& Gaginskaya (1984) and Hutchinson (1987). Since 2005 they have become established as a new model in avian cytogenetics (Schmid et al. 2005).

Additionally, LBCs are used as model structures in studies of transcriptional regulation, as their transcription activity is visible even under a light microscope. Transcriptional activity analyses are performed based on the assumption that side loops of LBCs are the active sites. 
Reduction of transcriptional activity corresponds with smaller sizes of side loops (Varley et al. 1980, Jamrich et al. 1983, Scheer et al. 1984, Baldwin \& MacGregor 1985, Callan et al. 1987, Scheer 1987, Gaginskaya \& Tsvetkov 1988, Gall \& Murphy 1998, Morgan 2002, 2007, Gaginskaya et al. 2009).

In transcriptionally active regions of $L B C s$, histone proteins give place to non-histone proteins, inducing the loss of the nucleosome structure of coding chromosome segments which assume the shape of a loop. However, the exposure of nucleosomes, which enables DNA transcription, does not cause histone dissociation but leads to a kind of spatial rearrangement of the transcribed regions, allowing the entry of RNA polymerase into the promoter sequence. This occurs through the fixation of regulatory proteins at the site of a remote activating sequence. A specific characteristic of lampbrush chromosomes is that they become incorporated in the structure of the chromatin of non-histone proteins (HMG - High Mobility Group). HMGs are structural proteins of chromatin. They reduce its condensation (Hill et al. 1974, Di Mario et al. 1989, Korner et al. 2003). In transcriptional units with insignificant or declining transcription, nucleosomes are present in the axial part of the chromosome between sets of polymerase units. The gaps between the polymerase blocks are not even. After polymerase has passed along the DNA matrix and the regulation proteins have become dissociated, nucleosome structure formation follows (Spring \& Franke 1981, Gaginskaya et al. 2009).

Despite the fact that increasingly more studies are devoted to $L B C s$, these structures remain largely unexplored. Neither the exact mechanism of $L B C$ formation out of little mitotic chromosomes, nor the way lampbrush chromosomes function in the oocyte are known. Nevertheless, the scale and form of transcription of these chromosomes suggest that this may be the earliest form of »looking after« a potential embryo (Rodinov 1996).

The aim of the present paper was to compare the structure of lampbrush chromosomes isolated prior to and after the reproductive period of the European domestic goose (Anser anser).

\section{Material and methods}

Lampbrush chromosomes were prepared by manual isolation from oocytes with diameters of 1.5-3.5 mm obtained from sexually mature geese using the standard procedure (MacGregor \& Varley 1988) modified for bird oocytes (Solovei et al. 1992, Rodinov \& Chechik 2002). The preparations were stained with Coomassie Blue R250. The first five macrobivalents and lampbrush sex bivalents (sex LBCs) were identified on the basis of their length and marker structure determined for avian lampbrush chromosomes (Solovei et al. 1993, Mizuno \& MacGregor 1998). The cytological map of Gallus domesticus LBCs described by Chelysheva et al. (1990), Rodinov et al. (2002) and Schmid et al. (2005) was particularly useful.

The preparations were analysed under an Olympus BX 50 light microscope (Olympus, Tokyo, Japan). A $100 \times$ zoom was used to identify specific marker structures, while the whole bivalent was examined with a $20 \times$ zoom. A detailed imaging analysis was carried out using the multiscan image analysis system, the karyotype software and graphic software compatible with the system. The analysis of transcriptional activity concerned the position and structure of the side loops, as well as the location of chiasmata and protein bodies in respective bivalent pairs. The pre-reproduction bivalents were marked with lowercase "a« and the post-reproduction ones with lowercase $» b$ «. The marker structures of the bivalents were successively numbered. 


\section{Results}

The LBCs sampled before the reproduction featured regions with well-preserved side loops (active transcription areas) alternating with regions with reduced side loops (transcriptionally inactive areas). Specific marker structures (distinctive side loops) were identified on the LBCs. After the egg production season, the LBCs had the characteristic atrophic side loops. What could be clearly seen were the chromomeres, visible as dark lumps distributed over the whole length of the chromosome. The following marker structures were identified in the LBCs under analysis: giant lumpy loops (GLLs), marker loops (MLs), distal boundary loops (DBLs), proximal boundary loops (PBLs), telomeric loops (TLs), telomeric bow-like loops (TBLs), telomeric lumpy loops (TLLs), double bridges (DBs), chiasmata (Chs) and protein bodies (PBs).

\section{Comparison of the structure of $L B C S 1$ prior to and after the reproductive period}

Seven groups of marker loops (MLa1-MLa7), Ta telomeric loops in the distal segment and TBLa telomeric loops were identified in the bivalent sampled before the egg production period. Chromatin without the loops, limited by the DBLa and PBLa markers delineates the bivalent centromere. Additionally, seven chiasmata (Cha1-Cha7) and two protein bodies (PBa1 and PBa2) connected with each of the bivalent homologues (Figure 1a) were spotted on the bivalent. Remnants of six groups of marker loops (MLb1-MLb6), telomeric loops (Tb) in the distal segment and already fragmented TBLb loops were identified on the bivalent sampled after the egg production period. The area adjacent to the DBLb1 and PBLb1 markers was identified as a centromere of the bivalent. Additionally, four chiasmata (Chb1-Chb4) and protein bodies (PBb1 and PBb2) connected with each of the bivalent homologues (Figure 1b) were found on the bivalent.

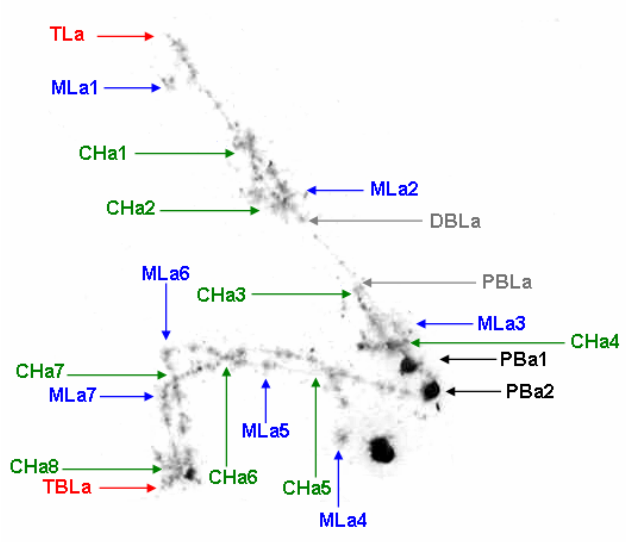

$50 \mu \mathrm{m}$

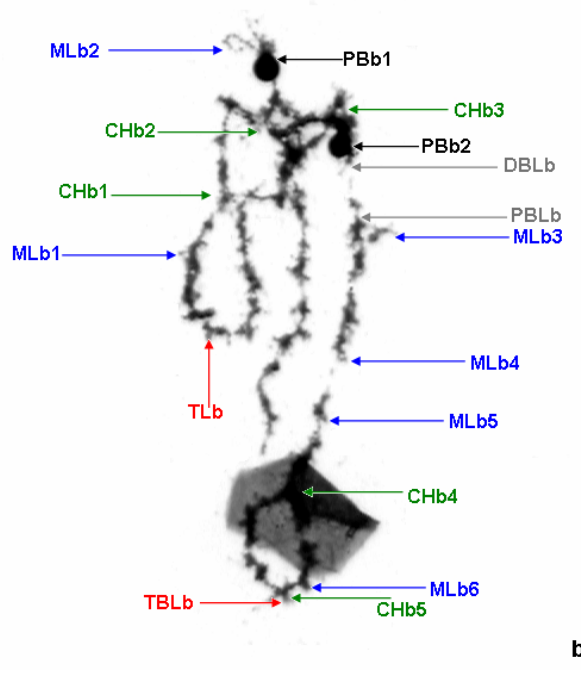

Figure 1

Comparison of the structure of LBC 1 prior to (a) and after (b) the reproductive period. Arrows indicate marker structures of bivalents. 


\section{Comparison of the structure of $L B C s 2$ prior to and after the reproductive period}

Six groups of marker loops (MLa1-MLa6), telomeric loops (Ta1 and Ta2), as well as TLLa1 and TLLa2 loops located in the terminal regions were identified in the bivalent. The segment without the loops, encompassed by the DBLa and PBLa markers is a bivalent centromere. Moreover, protein structures (PBa1 and PBa2) connected with each of the homologues and five chiasmata (Cha1-Cha5) were detected on the bivalent. Figure $2 \mathrm{~b}$ depicts the second LBC obtained after the reproductive period. Residual marker loops (MLb1-MLb5), telomeric loops (Tb1 and Tb2) and well-preserved TLLb1 and TLLb2 loops were detected on the bivalent. The bivalent centromere was spotted in the loopless segment between the DBLb and PBLb markers. In addition, small protein bodies connected with each of the homologues (PBb1 and $\mathrm{PBb} 2$ ) were identified. Five spots likely to contain chiasmata (Chb1-Chb5) were also found on the bivalent.

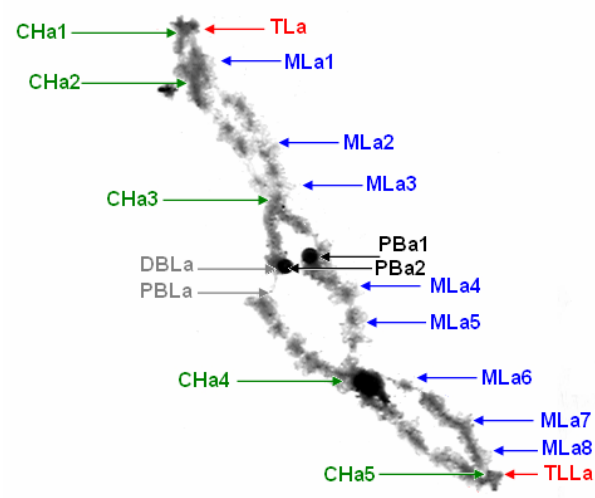

$50 \mu \mathrm{m}$

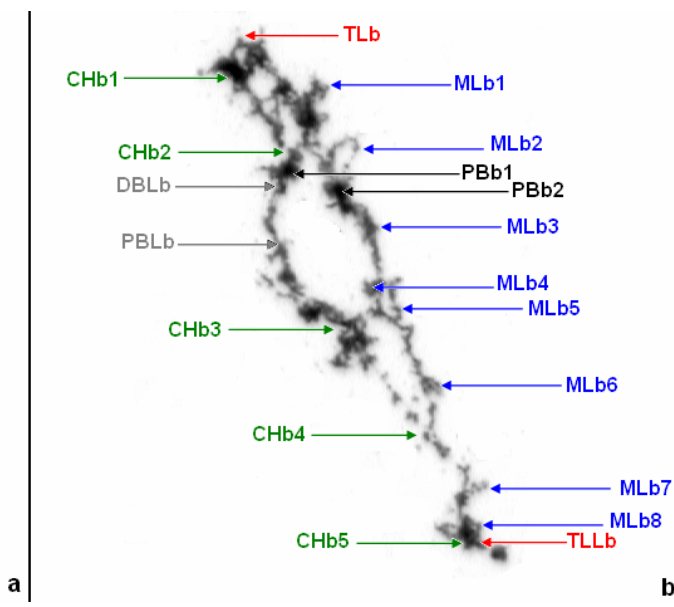

Figure 2

Comparison of the structure of $L B C 2$ prior to (a) and after (b) the reproductive period. Arrows indicate marker structures of bivalents.

\section{Comparison of the structure of $L B C s 3$ prior to and after the reproductive period}

Very prominent clusters of marker loops (MLa1 and MLa2), as well as large GMLa loops were identified on the bivalent. In the distal part of the bivalent there are Ta loops that, along with TLLa loops form the distinct "prongs«. Two chiasmata (Cha1 and Cha2) spots were found on the bivalent. In the proximity of Cha2 there are small TBLa loops. Numerous protein bodies (PBs) were identified close to the bivalent. Figure $3 \mathrm{~b}$ depicts the third LBC sampled after the reproduction period. The bivalent is almost completely devoid of side loops. Marker structures MLb1 and MLb2 are present as large chromomeres, while the GMLb structures have retained the shape of large and conspicuous loops. Moreover, well-preserved TLLb loops were also observed on the bivalent. The Tb loops also had the shape of distinctive "prongs", whereas the TBLb loops had the open form that is so uncommon in lampbrush autosomes. Furthermore, well-preserved chiasmata locations (Chb1 and Chb2) were observed on the bivalent. Protein bodies (PBs) were identified close to the bivalent. 

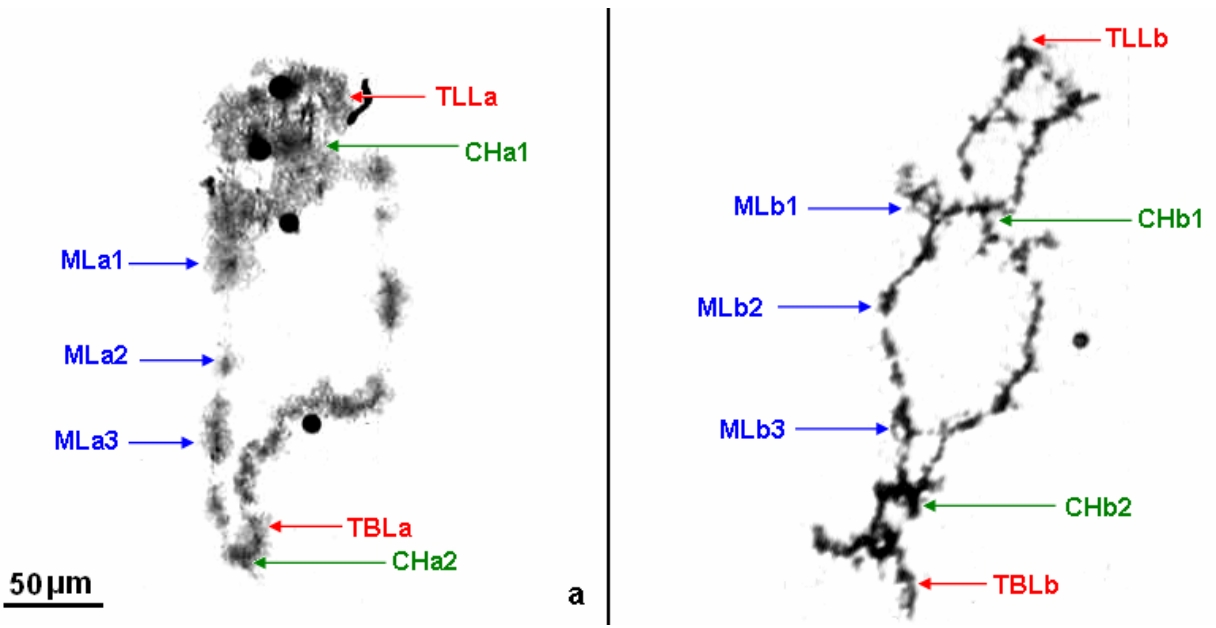

b

Figure 3

Comparison of the structure of $L B C 3$ prior to (a) and after (b) the reproductive period. Arrows indicate marker structures of bivalents.

\section{Comparison of the structure of $L B C S 4$ prior to and after the reproductive period}

Three groups of marker loops (MLa1-MLa3) and the distinct TBLa loops were identified on the bivalent. In the proximity of MLa3 there is a unique structure that was not observed in the other bivalents. This DB structure is a large »loopless« area, parallel in both chromatids of the bivalent. Additionally, two chiasmata (Cha1 and Cha2), as well as numerous bivalentspecific protein bodies (PBa1-PBa5) were found on the bivalent. Figure $4 \mathrm{~b}$ depicts the fourth LBC sampled after the egg production period had ended. Remnants of two groups of marker loops (MLb1 and MLb2), very prominent TBLb loops and telomeric Tb loops were identified on the bivalent. In the proximity of one of the marker loops there is a DB. Two chiasmata (Chb1 and Chb2), as well as two bivalent-specific protein bodies: a large (PBb1) and a small one (PBb2) were identified on the bivalent.
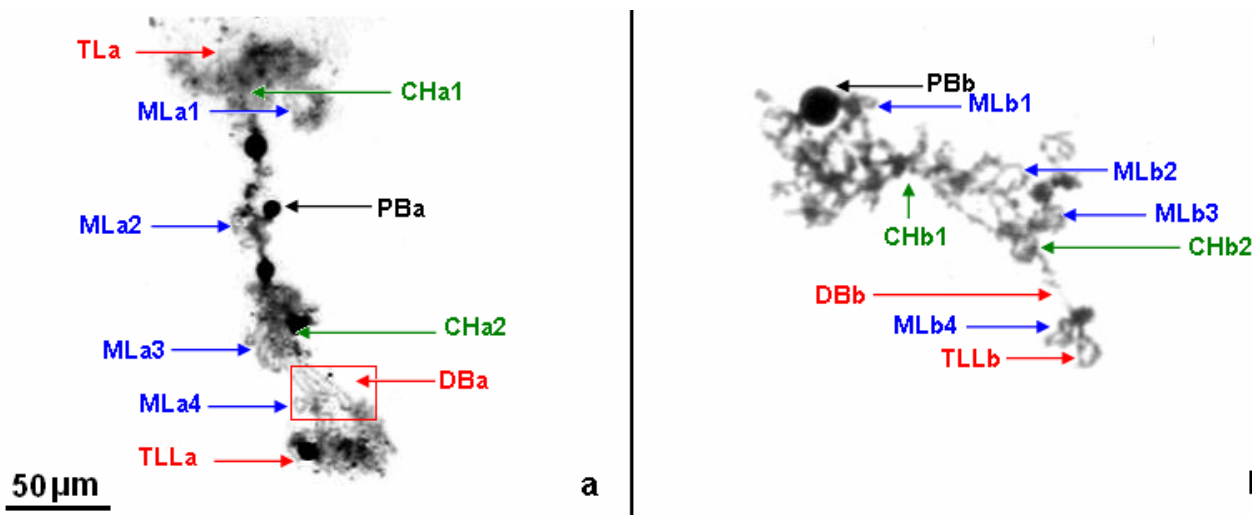

Figure 4

Comparison of the structure of LBC 4 prior to (a) and after (b) the reproductive period. Arrows indicate marker structures of bivalents. 


\section{Comparison of the structure of $L B C s 5$ prior to and after the reproductive period}

Two clusters of marker loops (MLa1 and MLa2), TBLa loops and two chiasmata (Cha1 and Cha2) were identified on the bivalent. Figure $5 b$ depicts the fifth $L B C$ isolated from the oocytes after the reproductive period. Remnants of two clusters of marker loops (MLb1 and MLb2) and two chiasmata (Chb1 and Chb2) are visible in the bivalent. Telomeric TBLb loops were identified in the distal part of the bivalent.

$50 \mu \mathrm{m}$

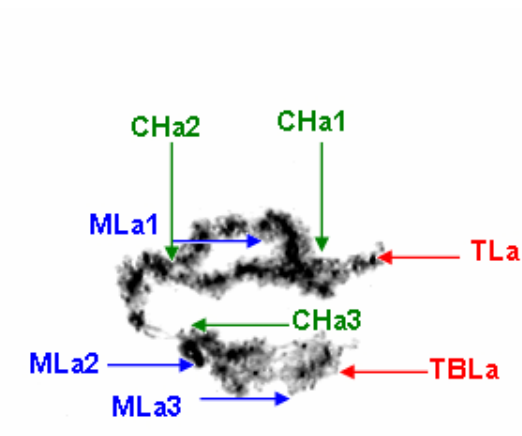

Figure 5

Comparison of the structure of LBC 5 prior to (a) and after (b) the reproductive period. Arrows indicate marker structures of bivalents.

\section{Comparison of the structure of the sex $L B C$ s prior to and after the reproductive period}

TBLa and TLa structures connected with the free telomere of the $Z$ chromosome were identified in the LBC sex ZWa. A GLLa loop was observed near the chiasm linking chromosomes $\mathrm{Z}$ and $\mathrm{W}$. Such loops are very distinctive and easy to spot even on bivalents with a dense mantle of loops. Over the entire length of the $Z$ chromosome, in specific places there are clusters of marker loops (MLa1-MLa3). In the vicinity of loop MLa3 small protein bodies were identified (PBa1). Structure of chromosome $W$ in which all the marker structures are in the form of large side loops; chromosome $W$ is present as a heterochromatic lump (Figure 6a). TBLb loops connected with the free telomere of chromosome $Z$ were also identified on the post-reproduction sex LBCs. In the proximity of the TBLb loop a PBb1 was observed. Three clusters of marker loops (MLb1-MLb3) were observed in the Z chromosome. The same as in the pre-reproduction bivalents, the GLL structure is located close to the chiasm linking chromosomes $Z$ and $W$. After the egg production period, the GLL structure assumes the shape of very large loops with an open structure. As in the prereproduction bivalents, the structure of chromosome $W$ depends on the structure of the entire sex bivalent. In bivalent $b$, the loops of chromosome $W$ are withdrawn towards the axis of the chromosome (Figure $6 b$ ). 


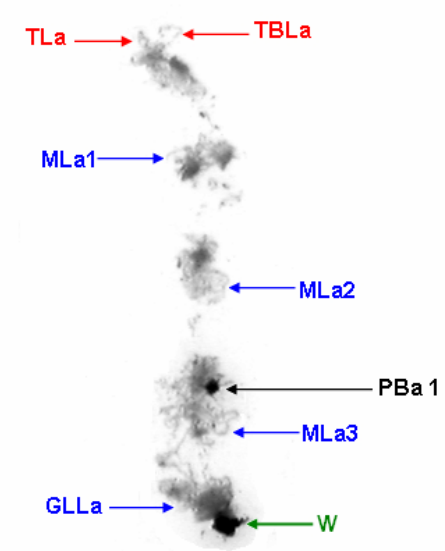

$50 \mu \mathrm{m}$ a

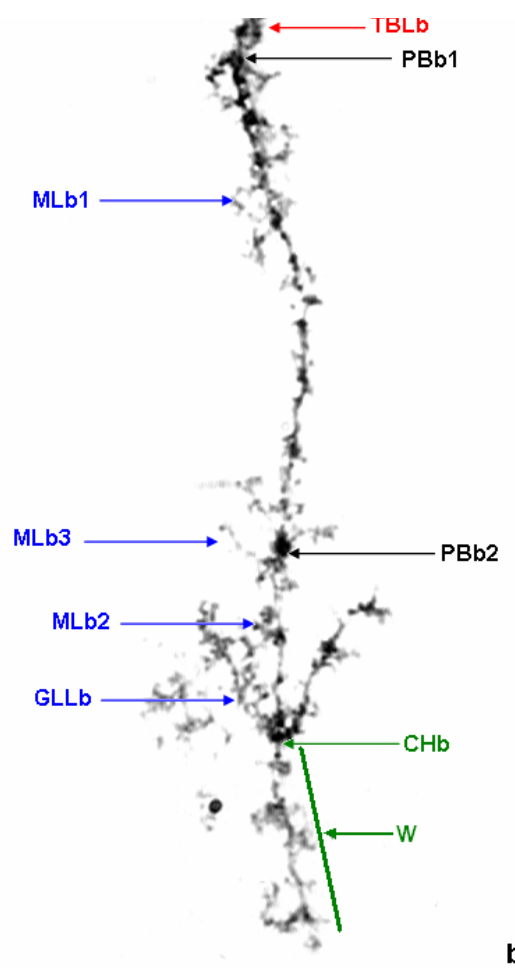

Figure 6

Comparison of the structure of the sex LBC prior to (a) and after (b) the reproductive period. Arrows indicate marker structures of bivalents.
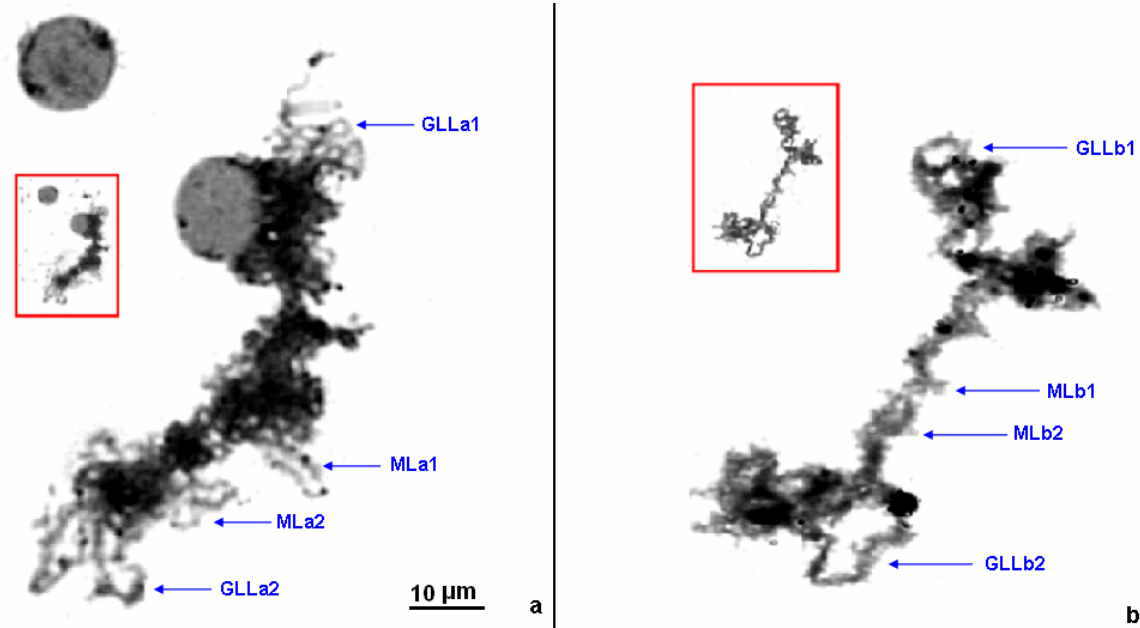

Figure 7

Comparison of the structure of the micro-LBC prior to (a) and after (b) the reproductive period. Arrows indicate marker structures of bivalents. 


\section{Comparison of the structure of the micro $L B C$ s prior to and after the reproductive period}

The analysis of the structure of microbivalents is possible only at a 100 -fold zoom. For a better comparison of the sizes of macro- and microbivalents, the microbivalent images at the $20 \times$ zoom were inserted as frames within Figure $7 \mathrm{a}$ and $7 \mathrm{~b}$. This is the zoom at which the first five macrobivalents and the sex bivalent were analysed.

Figure 7a depicts a sample microbivalent isolated from goose oocytes before the reproductive period. Along the whole axis of the microbivalent there is a mantle of side loops, large marker loops (MLa1 and MLa2) and characteristic telomeric loops GLLa1 and GLLa2. Figure $7 \mathrm{~b}$ depicts a sample goose microbivalent obtained after the egg production period. Over the whole length of the bivalent, the side loops are withdrawn towards the axis and are present in the form of chromomeres. Only the marker loops (MLb1 and MLb2) are well-preserved. In the distal parts of the microbivalent there are the gigantic GLLb1 and GLLb2 loops.

\section{Discussion}

The available bibliography did not contain any publications analysing the structure and transcriptional activity of goose LBCs or those of any other water poultry species. Moreover, this is the first study comparing the structure of pre- and post-reproduction LBCs. Therefore, the results of the study were compared with the results of experiments analysing the structure of LBCs in hens, quails, turkeys, doves, chaffinches, sparrows and amphibians.

The first goose $L B C$ is characterised by a relatively high number of chiasmata and marker loops. These structures remain well preserved on the bivalent after the egg production period. The difference concerns the whole mantle of $L B C$ side loops that recede towards the axis of the chromosome after the egg production. There are also telomeric loops (TBL) on the LBCs both prior to and after reproduction. Such loops had also been observed on the first LBC in hens (Hutchison 1987, Chelysheva et al. 1990, Solovei et al. 1992, 1993, 1995, Rodinov et al. 2002, Schmid et al. 2005) and chaffinches (Saifitdinova et al. 2003). On the other hand, they had not been identified on the first LBC of the quail (Rodinov \& Chechik 2002, Schmid et al. 2005). What is interesting is the significance and location of PBs. These structures are present in cells only in association with LBCs and their role has not been entirely explained (Morgan 2002, 2007). In the first goose bivalent, two protein bodies (PBs) connected with each homologue of the bivalent were identified. PBs were observed on the chromosomes both prior to and after the egg production period. PBs are not typical of the LBCs of all avian species. Apart from geese, they have only been identified in chaffinches, doves and sparrows (Solovei et al. 1993, Saifitdinova et al. 2003, Krasikova et al. 2004).

The second lampbrush chromosome was characterised by a high number of chiasmata and ML loops. The configuration of ML loops was preserved on the bivalent sampled from the geese after reproduction but the number of chiasmata was reduced. The reduction in the number of chiasmata is probably due to their terminalisation. In the region limited by the DBL and PBL markers there are two protein bodies associated with each homologue of the bivalent. The spot is identified as a centromere of the bivalent. The structure of this segment is preserved in the LBC after the egg production period, as well. The same configuration of PBs was observed in chaffinches (Saifitdinova et al. 2003). On the other hand, PBs were 
not identified in LBCs of hens, quails and turkeys (Chelysheva et al. 1990, Solovei et al. 1992, Myakoshina \& Rodinov 1994, Rodinov \& Chechik 2002, Schmid et al. 2005). In the second goose bivalent no telomeric TBL loops were found. These are typical of the second LBC in hens (Chelysheva et al. 1990, Solovei et al. 1992, Schmid et al. 2005).

On the third goose LBC, two chiasmata and three marker loop $(\mathrm{ML})$ clusters were identified. Some of the loops were classified as GLL - large marker loops. They were found near the first chiasm, in the analogical bivalent spots. Large GLL loops are also typical of the third bivalent of other bird species mentioned above but in no other species are they as large as in geese. The GLL loops are particularly well visible in the pre-reproduction goose bivalent on which the mantle of side loops and other marker loops are withdrawn towards the chromosome axis. Characteristic telomeric T loops, located in the telomere adjacent to the first chiasm, were observed in the post-reproduction LBC. The loops were folded into the centre of the bivalent and formed the distinctive »prongs«. Such loops were not observed in LBCs of other bird species. Probably, this distinctive configuration is likely to be observed in LBCs after the egg production period, when the other marker and telomeric loops are inactive. In the opposite telomere, open TBL loops were identified. TBL loops had been observed only on the third bivalent of chaffinches (Saifitdinova et al. 2003).

Apart form the ML marker loops and numerous protein bodies, the fourth bivalent isolated from the pre-reproduction geese contained the DB structure that was not observed in other goose LBCs. The DB structure remains well preserved on the bivalent after the egg production period. Sizeable DBs had only been identified in the fourth chromosome of hens (Schmid et al. 2005). Small DBs had been observed on the LBCs of chaffinches (Saifitdinova et al. 2003).

The same as in hens (Schmid et al. 2005), the fifth goose bivalent had the distinctive TBL loops. The change of bivalent structure after the egg production period as compared to its pre-reproduction state corresponded with the commonly accepted assumption that postreproduction bivalents do not have the mantle of side loops but only marker structures that are the last to become degraded.

The sex bivalent of birds has the shape of an asymmetric univalent. One of the telomeres, in the form of a compact knob, is considered as a condensed microchromosome W (Hutchinson 1987, Mizuno \& MacGregor 1998). As for the sex bivalent of geese, its various forms were observed both prior to and after the reproductive period. Diverse shapes and lengths of the ZW bivalent had also been described in other domestic birds: hens, quails, turkeys and doves (Solovei et al. 1993, Mizuno \& MacGregor 1998, Schmid et al. 2005) and wild ones: sparrows and chaffinches (Solovei et al. 1993, Saifitdinova et al. 2003). The distinctive TBL structures identified on the sex LBCs of geese prior to the egg production period and present in the free telomere of chromosome Z had otherwise been observed only in hens (Solovei et al. 1993; Mizuno \& MacGregor 1998). These loops are not present on the ZW bivalent of the quail, turkey, dove and chaffinch (Solovei et al. 1993, Mizuno \& MacGregor 1998, Saifitdinova et al. 2003).

Another distinctive structure in lampbrush sex bivalents are the large GLL loops situated in the region of the chiasm linking chromosomes $Z$ and $W$. In goose LBCs, these loops are large and easy to identify but not as prominent as the giant GLLs in turkeys, sparrows and chaffinches. In quail chromosomes, the structure was not observed. In turn, on the sex 
bivalents of hens the GLL structure can assume forms ranging from intensively condensed small loops to enormous loops as large as $1 / 3$ of the total length of the bivalent. These loops can have a closed (the distinctive loop) or open structure (Solovei et al. 1993, Mizuno \& MacGregor 1998, Saifitdinova et al. 2003).

On the sex LBCs, one PB associated with chromosome $W$ was always observed; the other PBs were present in various locations in the bivalent and in different numbers. Protein bodies had not been observed in sex LBCs of hens, quails and turkeys. On the other hand, PBs had been detected in regular locations on sex bivalents of the dove, sparrow and chaffinch. In the species mentioned above, one PB is always associated with chromosome W. Moreover, in doves, sparrows and chaffinches, PBs are present in the centromere segment of the sex bivalent (Solovei et al. 1993, Saifitdinova et al. 2003, Krasikova et al. 2004). A similar configuration of PBs was observed in the sex LBCs of geese. ML loops in the goose sex LBCs were found in different numbers and sizes. No marker loops were identified within the W chromosome in the geese. Such loops are typical of the bivalents of the quail and dove (Solovei et al. 1993, Saifitdinova et al. 2003).

Particularly interesting are the sex LBCs after the reproductive period. Very well preserved TBL and ML loops remain on the bivalents. The same as in the case of the pre-reproduction bivalents, two protein bodies (PBs) are associated with the two ML markers. In turn, on the post-reproduction bivalents close to the chiasm linking the $Z$ and $W$ chromosomes there are gigantic GLL loops. GLL loops were also observed in the geese prior to reproduction but they were not as well-developed. In hens, diversified forms of loops (GLL) had also been observed but they were sporadic on pre-reproduction chromosomes (Solovei et al. 1993). A distinctive feature of sex LBCs of the dove, chaffinch and sparrow are the enormous GGL loops present in the proximity of the chiasm linking the Z and W chromosomes (Solovei et al. 1993, Mizuno \& MacGregor 1998, Saifitdinova et al. 2003).

The different patterns of chromosomes sampled from pre- and post-reproduction geese are due to a modified transcriptional activity that is related to physiological processes in the organism and reflected in the morphological structure of the chromosome. The LBCs of prereproduction geese had the distinctive large side loops corresponding with transcriptional activity. The chromosomes sampled from the geese towards the end of the reproductive period had the characteristic small side loops and visible chromomeres resulting from the reduced transcriptional activity of the cell.

Regulation of lampbrush chromosome transcription is performed by means of modifications of chromosome structure and the activity of a number of post-transcription factors. The preservation of lampbrush chromosome structure during transcriptional activity is connected with the presence of the so-called »constitutive« nucleosomes (Spring \& Franke 1981, Gaginskaya et al. 1984, Scheer et al. 1984, Scheer 1987, Gaginskaya \& Tsvetkov 1988, Olins \& Olins 2003). The transcription of the oocyte-specific topoisomerase I (topo-I) variant is activated during the formation of lampbrush chromosome structures. This topoisomerase is present in lampbrush chromosome loops and participates in the spatial conformation of these structures. The inhibition of topo-I activity causes the lampbrush loops to recede and stimulates the condensation of nuclear chromatin (Gebauer et al. 1996, Morgan 2002, 2007).

The changes in gene expression commence at the moment of fertilisation (Davidson 1986). At this time, chromosome transcription activity almost completely disappears in 
most animal species until the first embryo divisions. In this period, chromosomes lose their distinctive lampbrush structure. Nuclear DNA transcription is resumed only after the embryo differentiation process has begun. The moment of DNA transcription reactivation is indeed a critical point in the development of the embryo, with the highest mortality rate. During this critical period for the developing embryo, protein synthesis goes on owing to the translation of mRNA accumulated in the oocyte (Landsberger \& Wolffe 1997). This means that an adequate number of ribosomes are necessary for the translation of the accumulated information and these are not produced by the embryo at the initial stage of its development. The effect of the amplification of rDNA genes is that the $18 \mathrm{~S}$ and $28 \mathrm{~S}$ ribosomal components become the most numerous population of the oocyte RNA (MacGregor \& Kezer 1970, Gaginskaya \& Gruzova 1975, Gaginskaya et al. 1984, Gaginskaya et al. 1988). This is particularly evident in birds, reptiles and amphibians whose oocytes accumulate an even 200 thousand times higher number of ribosomes than that of ribosomes in somatic cells. This may be associated with oviparity and the related worse conditions for embryo development than in the case of viviparous organisms. Though lacking the activity of their own nucleolar organizer regions, the zygotes and subsequently the embryos of these animals have to keep developing, which is made possible by the accumulated maternal ribosomes.

Lampbrush ribosomes also undergo seasonal changes that modify their morphology and transcriptional activity, which is particularly evident in hibernating amphibians. In the summer, when the animals are the most active, the activity of lampbrush chromosomes is the highest, as well. On the other hand, in the autumn, lampbrush chromosome activity abates. Nevertheless, this is not associated with morphological changes. At that time of the year, each transcription unit contains approximately 10 RNP filaments, while in the summer this number is twice as high. In the winter, transcription substantially declines. Both in chromosomes and in nucleoli numerous and very characteristic morphological changes take place (Tsvetkov \& Parfenov 1994) No more than $70 \%$ of the nuclear DNA is subject to transcription at the time. If in the case of physical factors, such as radiation, or numerous chemical factors a similar effect on the structure and activity of lampbrush chromosomes in various groups of animals can be expected, seasonal changes predominantly affect poikilotherms (Morgan 2002).

Lampbrush chromosomes are very dynamic structures in which the loops are formed and disappear in response to a number of nucleus-specific and environmental factors (Gall et al. 1999, Gall 2000, Doyle et al. 2002, Saifitdinova et al. 2003, Austin et al. 2009, Gaginskaya et al. 2009). The interrelation of active and inactive genes is controlled by epigenetic mechanisms operative at the level of DNA methylation and histone modification that change chromatin structure. The open conformation of chromatin is transcriptionally active, whereas the "closed" conformation is associated with the so-called transcription decline (Grummt \& Pikaard 2003).

The study of the influence of particular factors that improve or deteriorate hatching parameters is particularly interesting in the context of the poultry industry. This is due to the fact that the quality of the hatching material constitutes one of the most important aspects of poultry production economics. The influence of lampbrush chromosomes on hatching parameters seems unquestionable. The amount of mRNA that an oocyte produces on the maternal DNA matrix seems to be of critical importance for the development of the embryo whose autotranscription is initiated at a later stage. 


\section{References}

Austin C, Novikova N, Guacci V, Bellini M (2009) Lampbrush chromosomes enable study of cohesin dynamics. Chromosome Res 17, 165-184

Baldwin L, Macgregor HC (1985) Centromeric satellite DNA in the newt Triturus cristatus karelinii and related species: its distribution and transcription of lampbrush chromosomes. Chromosoma 92, 100-107

Callan HG, Gall JG, Berg CA (1987) The lampbrush chromosomes of Xenopus laevis: preparation, identification, and distribution of 5 S DNA sequences. Chromosoma 95, 236-250

Chelysheva LA, Solovei IV, Rodionov AV, Yakovlev A, Gaginskaya ER (1990) [Lampbrush chromosoms of the chicken: the cytological map of the macrobivalents]. Tsitologiia 32, 303-316 [in Russian]

Davidson EH (1986) Gene Activity in Early Development. 3rd ed. Academic Press, Orlando, FL, USA

Doyle O, Corden JL, Murphy C, Gall JG (2002) The distribution of RNA polymerase II largest subunit (RPB1) in the Xenopus germinal vesicle. J Struct Biol 140, 154-166

Gaginskaya ER, Gruzova MN (1975) [Detection of the amplified rDNA in ovarial cells of some insects and birds by hybridization in situ.] Tsitologiia 17, 1132-1137 [in Russian]

Gaginskaya ER, Tsvetkov AG (1988) [Electron microscopy research on the chromatin structure of dispersed lampbrush chromosomes in the hen]. Tsitologiia 30, 142-150 [in Russian]

Gaginskaya ER, Kropotova EV, Tsvetkov AG, Vengerov YY (1984) [Morphology of transcription in lampbrush chromosomes of avian oocytes.] Tsitologiia 26, 1064-1065 [in Russian]

Gaginskaya ER, Kasyanov VL, Kogan GL (1988) Amplification of ribosomal genes and formation of extrachromosomal nucleoli in oocytes of starfish Henricia hayashi (Asteroidea: Echinasteridae). Cell Differ 23, 53-60

Gaginskaya ER, Kulikova T, Krasikova A (2009) Avian Lampbrush Chromosomes: A Powerful Tool for Exploration of Genome Expression. Cytogenet Genome Res 124, 251-267

Gall JG (2000) Cajal bodies: the first 100 years. Annu Rev Cell Dev Biol 16, 273-300

Gall JG, Bellini M, Wu Z, Murphy C (1999) Assembly of the nuclear transcription and processing machinery: Cajal bodies (coiled bodies) and transcriptosomes. Mol Biol Cell 10, 4385-4402

Gebauer D, Mais C, Zinger K, Hock R, Lieb B, Scheer U (1996) Localization of a high molecular weight form of DNA topoisomerase I in amphibian oocytes. Int J Dev Biol 40, 239-244

Grummt I, Pikaard CS (2003) Epigenetic silencing of RNA polymerase I transcription. Nat Rev Mol Cell Biol 4, 641-649

Hutchison N (1987) Lampbrush chromosomes of the chicken, Gallus domesticus. J Cell Biol 105, 1493-1500

Jamrich M, Warrior R, Steele R, Gall JG (1983) Transcription of repetetive sequences on Xenopus lampbrush chromosomes. Proc Natl Acad Sci USA 80, 3364-3367

Krasikova A, Kulikova T, Saifitdinova A, Derjusheva S, Gaginskaya E (2004) Centromeric protein bodies on avian lampbrush chromosomes contain a protein detectable with an antibody against DNA topoisomerase II. Chromosoma 113, 316-323

Kropotova EV, Gaginskaya E (1984) [Lampbrush chromosomes from Japanese quail oocytes]. Tsitologiia 26, 1008-1014 [in Russian]

Landsberger N, Wolffe AP (1997) Remodeling of regulatory nucleoprotein complexes on the Xenopus hsp70 promoter during meiotic maturation of the Xenopus oocyte. EMBO J 16, 4361-4373

Macgregor HC (1986) The lampbrush chromosomes of animal oocytes, In: Risley MS (ed.) Chromosome Structure and Function, Van Nostrand Reinhold Publishing Corporation, New York, NY, USA, 152-186

Macgregor HC (1987) Lampbrush chromosomes. J Cell Sci 88, 7-9

Macgregor HC, Kezer J (1970) Gene amplification in oocytes with 8 germinal vesicles from the tailed frog Ascaphus truei Stejneger. Chromosoma 29, 189-206

Mizuno S, Macgregor HC (1998) The ZW lampbrush chromosomes of birds: a unique opportunity to look at the molecular cytogenetics of sex chromosomes. Cytogenet Cell Genet 80, 149-157 
Morgan GT (2002) Lampbrush chromosomes and associated bodies: new insights into principles of nuclear structure and function. Chromosome Res 10, 177-200

Morgan GT (2007) Localized co-transcriptional recruitment of the multifunctional RNA-binding protein CELF1 by lampbrush chromosome transcription units. Chromosome Res 15, 985-1000

Murphy C, Wang Z, Roeder RG, Gall JG (2002) RNA polymerase III in Cajal bodies and lampbrush chromosomes of the Xenopus oocyte nucleus. Mol Biol Cell 13, 3466-3476

Myakoshina YA, Rodionov AV (1994) [Meiotic lampbrush chromosomes in turkey, Meleagris gallopavo (Galliformes: Meleagrididae)]. Genetika 30, 577-583 [in Russian]

Olins DE, Olins AL (2003) Chromatin history: our view from the bridge. Nat Rev Mol Cell Biol 4, 809-814

Rodionov AV, Chechik MS (2002) [Lampbrush Chromosomes in the Japanese Quail (Coturnix coturnix japonica): Cytological Map of Macrochromosomes and Meiotic CrossING-over Frequency in Females]. Genetika 38, 1246-1251 [in Russian]

Rodionov AV, Lukina NA, Galkina SA, Solovei I, Saccone S (2002) Crossing Over in Chicken Oogenesis: Cytological and Chiasma-Based Genetic Maps of the Chicken Lampbrush Chromosome 1. J Hered 93, 125-129

Saifitdinova A, Derjusheva S, Krasikova A, Gaginskaya E (2003) Lampbrush chromosomes of the chaffinch (Fringilla coelebs L.). Chromosome Res 11, 99-113

Scheer U (1987) Structure of lampbrush chromosome loops during different states of transcriptional activity as visualized in the presence of physiological salt concentrations. Biol Cell 59, 33-42

Scheer U, Hinssen H, Franke WW, Jockusch BM (1984) Microinjection of actin-binding proteins and actin antibodies demonstrates involvement of nuclear actin in transcription of lampbrush chromosomes. Cell $39,111-122$

Schmid M, Nanda I, Hoehn H, Schartl M, Haaf T, Buerstedde JM, Arakawa H, Caldwell RB, Weigend S, Burt DW, Smith J, Griffin DK, Masabanda JS, Groenen MAM, Crooijmans RPMA, Vignal A, Fillon V, Morisson M, Pitel F, Vignoles M, Garrigues A, Gellin J, Rodionov AV, Galkina SA, Lukina NA, Ben-Ari G, Blum S, Hillel J, Twito T, Lavi U, David L, Feldman MW, Delany ME, Conley CA, Fowler VM, Hedges SB, Godbout R, Katyal S, Smith C, Hudson Q, Sinclair A, Mizuno S (2005) Second Report on Chicken Genes and Chromosomes 2005. Cytogenet Genome Res 109, 415-479

Solovei IV, Gaginskaya E, Allen T, Macgregor HC (1992) A novel structure associated with lampbrush chromosomes in the chicken, Gallus domesticus. J Cell Sci 101, 759-772

Solovei IV, Gaginskaya E, Hutchison N, Macgregor HC (1993) Avian sex chromosomes in the lampbrush form: the ZW lampbrush bivalents from six species of bird. Chromosome Res 1, 153-166

Solovei IV, Macgregor HC, Gaginskaya E (1995) Single stranded nucleic acid binding structures on chicken lampbrush chromosomes. J Cell Sci 108, 1391-1396

Spring H, Franke WW (1981) Transcriptionally active chromatin in loops of lampbrush chromosomes at physiological salt concentrations as revealed by electron microscopy of sections. Eur J Cell Biol 24, 298-308

Tsvetkov AG, Parfenov VN (1994) Seasonal transformations in the lampbrush chromosomes and the morphogenesis of the karyosphere capsule in Rana temporaria oocytes detectable by an analysis of the isolated nuclear structures. Tsitologiia 36, 64-70 [in Russian]

Correspondending author:

Katarzyna Andraszek

email: andrasz@uph.edu.pl

Department of Animal Genetics and Horse Breeding, Institute of Bioengineering and Animal Breeding, University of Life Sciences and Humanities, Prusa 14, 08-110 Siedlce, Poland 